\title{
SISTEMAS DE SIGNOS COMO FUENTES DE FORMACIÓN DE FRASEOLOGISMOS DEL ALEMÁN Y EL ESPAÑOL
}

\author{
Juan Pablo Larreta Zulategui
}

\begin{abstract}
This article tries to analyze a domain of phraseological investigation, such as the study of sources where phraseologisms (Phr) come from. For this purpose are used the german and spanish language and an contrastive analysis of both of them. Its specific character lies in the nature of the investigated sources, which are localized in signs systems and not out in the real world. Because of the origin of the mentioned sources, in this paper raise some questions concerning the sign-status of Phr-components, as soon as an argument about semantic features used often in linguistics to define lexical classes.
\end{abstract}

\section{Introducción}

\subsection{Sobre la fraseología general y contrastiva.}

La fraseología es una disciplina que, dentro de los estudios de lingüística occidentales, se ha desarrollado con especial vigor en el ámbito de la Germanística, debido fundamentalmente a la influencia de los estudios soviéticos sobre el tema a través de lingüistas de la extinta R.D.A. Al interés por cuestiones puramente teóricas durante los años setenta y parte de los ochenta, tales como la delimitación exacta del corpus fraseológico y la descripción de las estructuras semántico-formales de los fraseologismos (FR), le ha sucedido paulatinamente una tendencia al estudio de la fraseología contrastiva y aplicada a los campos de la didáctica, la traductología y la fraseografía.

La fraseología contrastiva es un paso necesario para los campos aplicados mencionados (en el campo fraseográfico, para la fraseografía bilingüe), y cuenta con un amplio abanico de posibilidades. Este extenso panorama se debe a dos causas, que son, por un lado, la complejidad formal y semántica del signo fraseológico y, por otro, la heterogeneidad de unidades lingüísticas que en un sentido amplio pueden considerarse fraseológicas. De este modo, FR pueden ser los fraseolexemas, los refranes, las estructuras con verbos de función, los esquemas fraseológicos, etc. Sólo un equipo de lingüistas puede acometer la tarea de comparar íntegramente los corpora de dos o más lenguas, incluso restringiendo éstos a una concepción estricta de la fraseología. ${ }^{1}$ Según mis conocimientos, únicamente existe un trabajo de este tipo entre lenguas europeas, realizado a partir de un proyecto iniciado en la

\footnotetext{
${ }^{1}$ Sobre la diferenciación entre fraseología en sentido amplio o estricto, cf. J. Häusermann 1977: 6-18.
} 
Universidad de Oulu (Finlandia), en el que han participado investigadores fineses y alemanes bajo la dirección de J. Korhonen. En esta obra se acomete el estudio contrastivo sistemático de los FR de las lenguas finesa y alemana, comparando estructuras formales y semánticas; textualmente, investigando el comportamiento textual del FR, sus modificaciones y posibles funciones pragmáticas; e incluso desde una perspectiva diacrónica, estudiando la historia de abundantes unidades fraseológicas. ${ }^{2}$

En esta contribución, me centraré en un aspecto parcial del análisis contrastivo, como es el estudio de las fuentes a partir de las cuales se generan las unidades fraseológicas. En este tema se supera habitualmente el campo estrictamente lingüístico, pues atañe a cuestiones referentes a la relación entre sistemas lingüísticos, comunidades lingüísticas y realidad circundante.

\subsection{Fuentes de formación de FR}

En un estudio sistemático sincrónico, además del contraste de las características estructurales de los FR, resulta interesante comparar las fuentes de las que beben estas unidades. No es necesario para ello aplicar una perspectiva diacrónica, realizando un estudio de la historia y evolución de los FR, pues en una investigación de este tipo se pretende constatar únicamente, a partir de la forma actual de las unidades fraseológicas, las afinidades y divergencias interlinguales en la relación de cada lengua con sus fuentes de formación de FR. Un estudio tal es interesante no sólo en sí, sino por estar relacionado con diversos aspectos del análisis cognitivo de los FR y con las transformaciones semánticas que pueden sufrir.

El interés en las fuentes de formación de FR se ha centrado en la investigación generalmente en los internacionalismos, FR presentes en diversas lenguas occidentales gracias al sustrato cultural común, representado sobre todo por la Antigüedad Clásica, la Edad Media y la Biblia. También el estudio de rasgos socioculturales propios, como diversos sistemas de monedas, pesas o medidas resultan de gran interés en tanto en cuanto son fuente antes de particularidades lingüísticas que de internacionalismos. Existen otros factores extralingüísticos no socioculturales estudiados en mayor o menor medida, tales como el mundo físico que rodea a una comunidad lingüística u objetos presentes en el día a día, como las prendas de vestir (cf. Földes, 1990; Klimaszewska 1991). Especialmente productivas son las partes del cuerpo humano o animal, que dan lugar a los denominados somatismos.

En este artículo, el análisis se centra en fuentes de formación de FR que hasta ahora, según mi información, han suscitado un interés menor en la investigación. Estas fuentes son los signos pertenecientes a los sistemas de comunicación lingüísticos oral y escrito (cf. apdos. 2 y 3), por lo que cabe establecer una diferencia básica respecto a las diversas fuentes mencionadas anteriormente, y es que éstas se hallan supuestamente dentro y no

\footnotetext{
${ }^{2}$ Los dos volúmenes de este trabajo, J. Korhonen (ed.), Studien zur Phraseologie des Deutschen und des Finnischen (Bochum 1995, 1996), están formados por una serie de contribuciones individuales que analizan todas las cuestiones mencionadas.
} 
fuera de la propia lengua (ese es el punto de partida que deberá ser reafirmado o negado en cada caso). Este enfoque da pie a realizar, en determinados casos, un análisis semiótico lingüístico de los componentes estudiados del FR como signos o signos lingüísticos; y en otros casos, a introducir un elemento de reflexión acerca de los rasgos semánticos empleados en la confección de las taxonomías de las clases léxicas. Ambos tipos de análisis se suman al estudio contrastivo.

La meta es, por tanto, investigar cómo la lengua se sirve de sí misma para generar otras unidades propias que la enriquezcan, en este caso con los ejemplos de las lenguas alemana y española. ${ }^{3}$ Con este propósito, se pueden establecer dos fuentes fundamentales para su análisis:

a) sistemas de signos de carácter gráfico, entre los que destaca el alfabeto como fuente principal, además de los signos ortográficos de puntuación y entonación (apdo. 2). ${ }^{4}$

b) palabras cuyo significado y/o función habitualmente se ha designado como "gramatical", "vacío", "relacional", "estructural”, etc., que adquieren teóricamente en el FR una nueva carga de significado (apdo. 3).

2. El alfabeto y los signos de puntuación y entonación: relaciones interlinguales, particularidades de transformación semántica y status semiótico de los componentes del FR.

\subsection{El alfabeto como fuente principal}

2.1.1 Se observa en ambas lenguas cómo el alfabeto se convierte en fuente de formación de FR a través de diversos mecanismos verificables en las dos lenguas, que encuentran así en las letras un cauce de enriquecimiento. Diversas equivalencias y disimilitudes interlinguales hacen sugerente el análisis detallado de los mecanismos mencionados en varias subdivisiones.

Por otro lado, se parte de la evidencia de que el lenguaje escrito no es sino una representación del oral. Por ello, desde una perspectiva semiótica, se considera que las

\footnotetext{
${ }^{3}$ Interesante sería también analizar la influencia de otros sistemas de comunicación no lingüísticos en la formación de FR. Destacan aquí los quinegramas fraseológicos, reflejo lingüístico de un signo de comunicación no gestual (v. gr., sich an die Stirn greifen/fassen y llevarse las manos a la cabeza), que tampoco han sido especialmente investigados (cf. Burger 1976); FR formados a partir de signos matemáticos o su verbalización (v. gr., ein Herr X, $j m$. ein X für ein U vormachen, in Null Komma nichts, gleich Null sein, Pi mal Daumen, stehen wie eine Eins, ser un cero a la izquierda, hacerse un siete); o FR formados a partir de elementos del sistema de signos musical (v. gr., das hohe $C$, dar el do de pecho).

${ }^{4}$ Curioso es, por otro lado, el reflejo de rasgos fonéticos en el lenguaje escrito, como observa agudamente Eismman en un FR del alemán, jm. s-tolpert mit der Zunge über'n s-pitzen S-tein, que representa de este modo la pronunciación del fonema /s/ como variante regional delante de oclusiva sorda en lugar del normativo /// (Eismann 1987: 232).
} 
letras del alfabeto responden aproximadamente a un modelo de signo sencillo, modelo documentado ya en el ámbito de la sintomatología médica griega del siglo III a. d. C., y que se centra en la relación temporal, causal o local de dos objetos o sucesos, de modo que uno es visto como signo del otro (Eschbach 1980: 44). Es una relación dual de sustitución que se observa también en el caso de las letras del alfabeto, que representan de este modo un fonema, en una relación 1:1 (sin considerar las asimetrías entre sonidos y letras que se dan en los sistemas lingüísticos). No es ciertamente una relación temporal, causal o local, sino arbitraria, pero responde a la misma idea de sustitución y por tanto equiparación directa de un objeto y su signo. Este modelo de signo cambiará, como se verá, en tanto en cuanto las letras se transforman en componentes internos de los FR.

2.1.2 Se hallan en primer lugar FR formados por letras cuya motivación se halla concretamente en la antigua idea de que en las letras y las palabras se halla el concepto al que se refieren, por lo que su dominio y conocimiento suponen la sabiduría en sí. En este contexto, encontramos el mismo motivo en ambas lenguas, referido al alfabeto como el compendio de todo conocimiento, en

$$
\begin{aligned}
& \text { jm. das } A B C \text { beibringen } \\
& \text { etw. gehört zum } A B C \\
& j m . \text { durchs ganze } A B C \text { loben }
\end{aligned}
$$$$
\text { conocer/saber algo como el abecé }
$$

Alfabetos ajenos al sistema lingüístico propio, por mor de la tradición cultural, contribuyen también a la formación de FR. Este hecho se observa en otro motivo, como son el primer y el último elemento del alfabeto convertidos en punto de partida de nuevos FR. Este motivo genera a veces equivalentes interlinguales, v. gr.,

\section{Das $A$ und $O$}

el alfa y la omega

aunque en este motivo se verifican un mayor número de FR en alemán sin equivalente en castellano, v. gr., von A bis Z, bis zum TZ/Tezzet.

Lo cierto es que las letras reciben un significado léxico que no tenían antes, eso sí, translaticio o idiomático, y dejan de cumplir esa función de sustitución en la relación dual anteriormente descrita. Este fenómeno de transformación semiótica de las letras del alfabeto al convertirse en componentes de FR se observa en unidades fraseológicas que poseen una estructura semántica composicional, es decir, una estructura semántica en la que es posible reconocer un significado en cada uno o, al menos, alguno de los componentes del FR, por lo que es más fácil y unívoco adscribirles ese significado léxico a las letras que 
forman parte de los mismos. Frente a estos FR composicionales se hallan FR de estructura no composicional, en los que a ningún componente del FR se le puede adscribir un significado individual, v. gr., jm. etw. aus dem Kreuz leiern (cf. Dobrovol'skij 1995: 28-29) o como quien oye llover. Se constata por tanto un cambio semiótico en las letras del alfabeto cuando se convierten en componentes internos del FR. Las letras dejan de ser el sustituto mecánico de un sonido, para convertirse en la representación de un concepto ligado a la aprehensión de la realidad extralingüística. Se convierten así pues en auténticos signos lingüísticos, ya sea según la concepción de de Saussure o de Ogden y Richards, según la mayor o menor atención prestada a la realidad extralingüística en la formación del signo linguístico. La concepción de estos autores del signo lingüístico dirige su atención, en el caso de Ogden y Richards, al problema de la relación entre pensamiento y lenguaje, excluyendo con razón a los elementos del alfabeto de la misma. Serébrennikov reafirma la diferencia entre las distintas concepciones del signo:

"Bei einer solchen Definition des Zeichens läßt sich freilich die menschliche Sprache in ihrem gesamten Umfang - sowohl das Inventar der lexikalischen und lautlichen Einheiten als auch die Modelle ihrer Verknüpfbarkeit, ja selbst die Buchstabenrepräsentation der Laute - in gleicher Weise leicht zur Kategorie des Zeichens rechnen. Eine solche Auffasung trägt jedoch wenig zur Klärung der semiotischen Natur der natürlichen Sprache bei, deren Zeichen, unmittelbar mit der psychischen Tätigkeit des Menschen, mit seinem Denken verbunden [27; 719; 822], diesem komplizierten, viele Funktionen ausübenden System große Eigenständigkeit und einmalige Spezifik verleihen." (Serébrennikov (ed.) 1973: 81).

En resumen, cualquiera que sea la acepción de signo lingüístico preferida, lo cierto es que la naturaleza de las letras del alfabeto cambia sustancialmente, gracias al significado idiomático o translaticio que obtienen dentro del FR.

Desde un punto de vista interlingual llama la atención, además de las equivalencias observadas de determinadas unidades, la ortografía dentro del FR en ambas lenguas, que difiere, siendo el español el que mejor señaliza el nuevo status de signo lingüístico de los componentes del alfabeto, ya que deja de representar las letras como tales, convirtiéndolas en palabras, significantes del modelo dual o trilateral del signo lingüístico, mientras que el alemán fluctúa entre ambas posibilidades, incluso en el mismo FR, v. gr., bis zum TZ/Tezzet. ${ }^{6}$

Respecto a la motivación analizada, ese valor mágico de las letras también se va diluyendo a lo largo de la historia según Eismann, para quien

"Mit der Schrift und dem Schreiben selbst waren im Verlaufe der Menschheitsgeschichte eine Reihe wichtiger Tätigkeiten verbunden, und nach der anfänglichen Mystifizierung der Schreiber und Schriftgelehrten erkannte man offentsichtlich (sic) bald den Unterschied zwischen Schrift, schriftlich Fixiertem und der jeweiligen Bezogenheit auf eine konkrete

\footnotetext{
${ }^{5}$ Se refiere aquí Serébrennikov a la relación dual de sustitución antes descrita.

${ }^{6} \mathrm{La}$ ortografía de estos FR es la tomada de los diccionarios consultados y no de otras posibles fuentes.
} 
Situation, den Unterschied zwischen Buchstaben und Geist, wie wir in der Bibel finden (2. Kor 3, 6) und worüber es in allen Sprachen viele phE gibt." (Eismann 1987: 232)

Esta diferenciación es apreciable en FR de ambas lenguas como der Buchstabe des Gesetzes, leere Buchstaben, sich nach den toten Buchstaben richten, dem Buchstaben nach erfüllen en alemán; o saberse algo al pie de la letra y letra por letra en español. En estos FR naturalmente no existe ningún tipo de transformación en la estructura de los signos que componen las unidades fraseológicas, ya que en ellos no están presentes las letras como tales.

2.1.3 También otros FR deben genéticamente su existencia al alfabeto, como son FR con letras como componentes internos en los que éstas representan abreviaturas. Ejemplos de esta fuente en alemán son etw. nach Schema F erledigen, Vitamin B, etw. aus dem Effeff/ff beherrschen/können/verstehen, da will ich ein großes $P$ vorschreiben; y en español irse a la eme, mandar a la eme a alguien. Es posible suponer un origen similar para los FR saber/explicar algo ce por be y de pe a pa, aunque no he encontrado ninguna explicación a su génesis. ${ }^{7}$ En cualquier caso, en el contraste interlingual, la unidad de pe a pa encuentra un equivalente en un FR alemán señalado anteriormente como es von $A$ bis $Z$.

En lo referente a la cuestión semiótica, se observa en estas unidades exactamente el mismo fenómeno que anteriormente, esto es, la transformación en signo lingüístico de la letra como componente del FR. En estos casos, no obstante, ese cambio se produce de un modo más directo o evidente, ya que la abreviatura indica la sustitución de una palabra, gracias a lo cual la letra adopta el status de ésta. ${ }^{8}$

Interlingualmente se observa en estos casos la total falta de equivalencias, a diferencia del subapartado anterior. Puede suponerse que las equivalencias anteriores se fundaban en una creencia supralingüística, en una consideración de origen cultural no limitada a una comunidad lingüística. Ahora, sin embargo, cada lengua muestra su propia evolución, ya que los FR no responden a conceptos o esquemas mentales universales. Ortográficamente, el español sigue determinando con mayor claridad el nuevo status semiótico de las letras, mientras que en alemán nos volvemos a encontrar a veces ante un proceder vacilante, v. gr., etw. aus dem Effeff/ff beherrschen/können/verstehen.

Reseñable en estos casos es el mecanismo eufemístico que se encierra a veces en estos FR, v. gr., Vitamin B o mandar a alguien a la eme, mecanismo que no es en absoluto ajeno a la fraseología, que se sirve igualmente de otros elementos estructurales como los nombres propios (cf. Földes: 1987: 13) y algunos pronombres personales como componentes internos (cf. Larreta: 1996) como base para la formación de expresiones eufemísticas en fraseología.

\footnotetext{
${ }^{7}$ Sobre las dificultades que plantea averiguar el origen de numerosos FR, cf. Iribarren 1994: 56-57, acerca de la unidad de pe a pa.

${ }^{8}$ Un caso especial lo constituye el FR para más/mayor inri, en el que las iniciales de Iesus Nazarenus Rex Iudaeorum se han lexicalizado hasta ser recogidas en el diccionario como una palabra.
} 
La referencia humorístico-eufemística se halla también en ein Engel mit einem B davor, si bien en este caso la letra conserva semióticamente su carácter.

2.1.4 La forma de algunas letras también motiva FR en ambas lenguas, ya sea con la misma base:

den Punkt auf das $i$ setzen

poner los puntos sobre las íes.

der Punkt/das Tüpfelchen auf dem $i$

o con una base distinta, que da pie a FR diversos en cada lengua, v. gr., jd. hat $O$-Beine, hacer eses, no saber hacer la 'o' con un canuto.

Igualmente la letra $l$ es motivo recogido en ambas lenguas, en las que se realiza una metáfora a partir de la analogía establecida entre el tamaño de la iota griega y el concepto de "mínimo, escaso, exiguo", según explican tanto Duden en el caso de los FR alemanes (Duden 1994: 366) como Iribarren en el caso de los españoles (Iribarren 1994: 21, 136137):

\author{
nicht (um) ein Jota \\ (um) kein Jota \\ (auch) nur ein Jota
}

no saber ni jota

Para el caso en el que se verifica una equivalencia interlingual a partir del motivo de la letra $i$, J. M. Iribarren proporciona una explicación a su nacimiento del cual puede colegirse un transfondo cultural común, aunque el autor señalado sólo se refiera a la unidad española. Así, la adición de un acento o virgulilla por parte de los copistas al adoptarse los caracteres góticos, para evitar que dos íes seguidas se confundieran con una $u$, evolucionó hasta convertirse en el siglo XVI en unos simples puntos en algunos copistas, lo cual resultaba excesivamente perfeccionista para otros (Iribarren 1994: 75).

En el contraste se observa también cómo el origen de un particularismo alemán, mit jm. Fraktur reden, también hace referencia a la forma de las letras, aunque en este caso de todo un sistema gráfico, la llamada escritura gótica, que pervivió en Alemania hasta bien entrado el siglo XX. Naturalmente en este caso no se plantea ningún problema de tipo semiótico.

En conjunto, las divergencias y equivalencias interlinguales observables muestran que este procedimiento de formación de FR es tanto generador de particularidades lingüísticas como de equivalencias interlinguales.

En lo que se refiere a su status como signos, nos hallamos ante casos distintos de los encontrados hasta ahora. De esta manera, los FR den Punkt auf das $i$ setzen o poner los 
puntos sobre las ies poseen una estructura semántica no composicional, de manera que no es posible adscribir de modo inequívoco un significado propio a la letra en cuestión que forma parte del FR. Debido a ello, no se puede considerar que ésta alcance el mismo status o la misma estructura de signo lingüístico que las descritas en casos anteriores. Más bien, dentro de la imagen que sustenta al FR sigue manteniendo su valor habitual.

El FR español no saber hacer la 'o' con un canuto tiene una estructura composicional sólo parcialmente (aunque ello pueda parecer contradictorio), pues sí puede empezar a construirse el significado global del FR a partir del significado de los componentes no, saber y hacer, pero este significado analítico no es demostrable en los demás componentes, que únicamente en conjunto adquieren un significado unitario, la ' $o$ ' con un canuto $\rightarrow$ "nada". Al igual que en el caso anterior, la letra mantiene su valor habitual.

Por último, particulares son los casos de los FR O-Beine haben y hacer eses, ya que la posible interpretación de composicionalidad de sus estructuras semánticas concede de nuevo a las letras-componentes el status de signo lingüístico, pero en estos casos han recibido un significado translaticio determinado por el carácter icónico de la relación entre forma y contenido. ${ }^{9}$

2.1.5 Finalmente, un último recurso de formación de FR con las letras como motivo es la referencia indirecta a las mismas, que a menudo es utilizado de nuevo como procedimiento eufemístico, como sich auf seine vier Buchstaben setzen (Eismann 1987: 240).

En relación con este mecanismo de la referencia indirecta, hallamos en castellano, v. gr., la interpretación que el erudito José María Sbarbi mantiene para el FR mantenerse en sus trece, según la cual tal expresión proviene de la alusión a las trece letras de la palabra $d$-e-t$e-r-m-i-n-a-c-i-o ́-n$, explicación que Iribarren considera inadmisible y extravagante, al igual que otras similares que nos proporciona Sbarbi para FR como tener la cabeza a las tres, echarlo todo a doce o apúntate ocho (Iribarren 1994: 71-72).

Tampoco en estos FR es constatable ninguna cuestión reseñable de tipo semiótico.

\subsection{Los signos de puntuación y entonación}

Los sistemas de signos de puntuación y entonación aportan una menor cantidad de FR en ambas lenguas. En estos casos no existe discusión alguna acerca de su composición como signos, ya que en ningún momento se utilizan verdaderamente los signos de dichos sistemas, sino su designación (como ya se ha visto en otros FR aparecidos anteriormente).

En el contraste interlingual se observa la existencia de FR en ambas lenguas que tienen como motivación los signos de punto y coma, aunque sólo en un caso se verifica una sinonimia estructural y parcialmente ideográfica (rota por la presencia del adjetivo en el FR español), concretamente en el par comparado

\footnotetext{
${ }^{9}$ Entendido el concepto de icono según Peirce (Peirce 1993: 65-66)
} 
einen Punkt machen

ponerle punto final a algo

Otros FR con estos motivos son ohne Punkt und Komma reden, con puntos y comas, sin faltar una coma, ser punto y aparte. Entre estos FR se puede encontrar un caso peculiar de lo que podría considerarse un falso amigo. Se observa al contrastar ohne Punkt und Komma reden $\rightarrow$ "pausenlos reden" frente a sin faltar una coma $\rightarrow$ "sin faltar/olvidar detalle". Las imágenes opuestas de estas unidades (ohne Punkt und Komma $\neq$ sin faltar una coma) podrían hacer pensar que sus significados deberían ser justamente antónimos, sin que sea éste el caso, en parte debido a la influencia semántica del verbo alemán que obligatoriamente aparece en el FR, que dirige el significado de la imagen de un modo específico.

Otros motivos son particulares de cada lengua, v. gr., ein großes Fragezeichen hinter etw. setzen, in Klammern hinzufügen, aussehen/dastehen wie ein lebendiges Fragezeichen, en alemán; entre comillas en español. Reseñable es el componente icónico de la transformación semántica operada en el FR aussehen/dastehen wie ein lebendiges Fragezeichen $\rightarrow$ "in schlechter Körperhaltung dastehen, eine unglückliche Figur machen", similar a la de FR ya analizados anteriormente y genéticamente distintos, como $O$-Beine haben y hacer eses.

\section{La lengua dentro de la lengua}

\subsection{El discurso indirecto en los $\boldsymbol{F} \boldsymbol{R}^{10}$}

3.1.1 La existencia de un discurso indirecto dentro de determinadas unidades fraseológicas demuestra cómo la lengua se sirve en cierto modo de sí misma para seguir creciendo. Es este mecanismo el que nos permite utilizar sin duda la expresión de "la lengua dentro de la lengua", ya que los mecanismos que serán descritos en los apartados posteriores (3.2 y 3.3) revelan en su caso la dificultad de utilizar tal expresión.

3.1.2 De esta manera, FR como tener algo/alguien un no sé qué o ser el no va más contienen auténticas oraciones que pueden ser construidas en el discurso libre de la propia lengua, y que adquieren un significado idiomático dentro de la unidad fraseológica, que utiliza para ello recursos gramaticales irregulares. Estas oraciones se utilizan así en forma de discurso indirecto dentro del FR, a partir de su hipotético uso en el discurso libre. En los ejemplos dados, las oraciones "no sé qué" y "no va más" están substantivadas, con los significados respectivos de $\rightarrow$ "rasgo difícil de definir" y $\rightarrow$ "el/lo mejor en algo".

El FR no decir 'esta boca es mía' representa un mecanismo un tanto especial respecto a los anteriores, pues el componente intralingüístico está señalizado ortográficamente por

\footnotetext{
${ }^{10} \mathrm{El}$ término discurso indirecto se utiliza aquí con una cierta libertad, ya que no se refiere a la repetición de un discurso pronunciado por un tercero o por uno mismo con anterioridad, sino a un hipotético discurso libre que es repetido en el interior del FR.
} 
medio de las comillas, con lo cual se transforma en discurso directo, como oración que puede ser construida en el discurso libre del idioma en cuestión. De este modo se percibe la actitud consciente de la comunidad lingüística de estar utilizando este mecanismo de la lengua dentro de la lengua. Esta consciencia metalingüística es del mismo modo evidente en el FR alemán wenn das Wörtchen wenn nicht wär' (wär' mein Vater Millionär), en el que el valor condicional de la preposición wenn es transformado en un significado idiomático.

Un caso original lo representa el FR andar con dimes y diretes, en el que los componentes que podrían considerarse de uso indirecto se han lexicalizado, adoptando la forma de un substantivo, por lo que ese uso indirecto queda solapado o cada vez más difuminado.

La presencia del verbo sagen/decir como núcleo de un FR verbal determina igualmente en cierto modo el uso indirecto de la lengua dentro de los FR, que puede así referirse claramente a actos de habla reconocibles en el exterior de las unidades fraseológicas. Ejemplo de este fenómeno es el par comparado

nicht nein sagen können no saber decir no

El mismo caso se da a través del uso de vocablos pertenecientes a otras lenguas. El acervo cultural común es el que provoca la equivalencia:

zu allem ja und amen sagen

Decir u. p. amén a algo"11

En alemán se encuentra un buen ejemplo de este uso indirecto de la propia lengua en una serie de FR que podrían denominarse con Fleischer esquemas fraseológicos (al. Phraseoschablonen), construcciones cuya inclusión en la fraseología es polémica y que se hallan en una zona intermedia entre la fraseología y la sintaxis. Fleischer los define como

"syntaktische Strukturen (...), deren lexikalische Füllung variabel ist, die aber eine Art syntaktischer Idiomatizität aufweisen. Das syntaktische Konstruktionsmodell hat eine vom entsprechenden nicht idiomatischen Modell abweichende, irreguläre Bedeutung." (Fleischer 1982: 136).

\footnotetext{
${ }^{11}$ Este uso indirecto de palabras extranjeras en FR también se encuentra sin el concurso del verbo decir/sagen. Es el caso del FR español ni el sursum corda. A veces, este mecanismo es difícilmente reconocible, pues algunas unidades son más opacas que otras y no dejan translucir claramente su motivación. Es el caso del FR berlinés jm. pomade sein, cuyo origen se sitúa según Duden en la expresión polaca po malu, y que a través de la etimología popular se transformó en la unidad fraseológica alemana correspondiente (Duden 1992: 553). De esta explicación se deduce que ese uso indirecto de la lengua en la formación de FR no tiene por qué ser siempre consciente.
} 
La serie a la que se hace alusión es la de los FR wer weiß was, wer weiß wen, wer weiß was für, wer weiß wie. La estructura léxico-sintáctica wer weiß + pronombre interrogativo tiene un significado idiomático aproximado de "unvorstellbar", expresado de modo irónico y/o peyorativo. Los componentes fijos del esquema fraseológico representan aquí ese discurso indirecto, ya que forman una posible oración del discurso libre, utilizada en su conjunto como componente de un FR.

\subsection{Palabras llenas y pallabras vacías de significado}

3.2.1 La afirmación de que existen palabras que como tales son fuentes de FR parece absurda e innecesaria por lo evidente de la misma, y efectivamente lo es sin una ulterior explicación, para lo cual estableceré una hipótesis de trabajo antes de analizar los distintos FR.

La estructura de la palabra como signo lingüístico, con su idea o concepto ligado al significante, es susceptible de sufrir una transformación semántica, v. gr. metafórica, por medio de la referencia a otro objeto de la realidad extralingüística con el que se establece una relación de similitud. Pero esa idea o concepto ligado al significante se funda en la relación del signo lingüístico con el mundo, con la situación, con el objeto, con la realidad extralingüística. En los casos de FR que a continuación se analizarán (FR en los que el núcleo semántico lo constituye generalmente una preposición o una conjunción), ese significado en el que se opera una transformación semántica no está ligado a la realidad extralingüística, sino a la lengua misma, o, al menos, esta aseveración podría defenderse, según mi opinión, a la vista de algunas teorías sobre clases léxicas desarrolladas en la lingüística, fundamentalmente en la formulación establecida por L. Tesnière. De esta manera, los FR así formados tendrían como fuente igualmente el propio sistema lingüístico.

3.2.2 Las abundantes disputas sobre las clases de palabras han generado distintas taxonomías, basadas generalmente en un conjunto de características, como lo muestra el cuadro de Duden (Tabla 1), en el que intervienen factores morfológicos, sintácticos y semánticos.

Una clasificación de este tipo es para Tesnière poco adecuada, pues no considera la preponderancia de unas características sobre otras:

"Eine brauchbare Klassifikation darf nämlich nicht offensichtlich verschiedene Kriterien zugleich verwenden. Man hat daher wesentliche von unwesentlichen Merkmalen oder, um in der Sprache der Logiker zu reden, übergeordnete von untergeordneten Merkmalen zu unterscheiden. Dieses Prinzip der Gewichtung der Merkmale führt uns zu einer Hierarchie der Kriterien, wobei das wichtigste zur Bildung von Klassen, das zweiwichtigste zur Bildung von Subklassen usf. dient." (Tesnière 1980: 62).

Sin entrar en la discusión sobre la mayor o menor validez de clasificaciones basadas en criterios heterogéneos y de las jerarquías que deben presidir las relaciones de dichos criterios $^{12}$, sí es del máximo interés en este trabajo el hecho de que Tesnière concede la

\footnotetext{
${ }^{12}$ En referencia a este problema, creo que es especialmente interesante la teoría ordenadora por medio de los
} 
preponderancia al aspecto semántico para distinguir dos clases de palabras fundamentales, "llenas" y "vacías". De este modo, este autor define así las denominadas palabras llenas de significado:

"Volle Wörter nennen wir die mit semantischer Funktion, also die, deren Ausdrucksform unmittelbar mit einer Vorstellung verbunden ist, die sie darzustellen bzw. hervorzurufen haben." (Tesnière 1980: 63).

\begin{tabular}{|c|c|c|c|}
\hline \multicolumn{4}{|c|}{ Merkmale } \\
\hline Wortart & Morphologisch & Syntaktisch & Semantisch \\
\hline Verb & $\begin{array}{l}\text { flektierbar: } \\
\text { Konjugation }\end{array}$ & $\begin{array}{l}\text { Funktion: v. a. Prädikat } \\
\text { Distribution: in Kongruenz mit dem } \\
\text { Subjekt (Personalform) }\end{array}$ & $\begin{array}{l}\text { Zustände, } \\
\text { Vorgänge, } \\
\text { Tätigkeiten, } \\
\text { Handlungen }\end{array}$ \\
\hline Substantiv & $\begin{array}{l}\text { flektierbar: } \\
\text { Deklination }\end{array}$ & $\begin{array}{l}\text { Funktion: Subjekt, Objekt, adverbiale } \\
\text { Bestimmung, Attribut } \\
\text { Distribution: mit Artikel }\end{array}$ & $\begin{array}{l}\text { Lebewesen, } \\
\text { Sachen (Dinge), } \\
\text { Begriffe } \\
\text { (Abstrakta) }\end{array}$ \\
\hline Adjektiv & $\begin{array}{l}\text { flektierbar: } \\
\text { Deklination } \\
\text { komparierbar }\end{array}$ & $\begin{array}{l}\text { Funktion: Attribut, adverbiale } \\
\text { Bestimmung } \\
\text { Distribution: } \\
\end{array}$ & $\begin{array}{l}\text { Eigenschaften, } \\
\text { Merkmale }\end{array}$ \\
\hline Artikel, Pronomen & $\begin{array}{l}\text { flektierbar: } \\
\text { Deklination }\end{array}$ & $\begin{array}{l}\text { Funktion: Attribut oder Substantiv- } \\
\text { Stellvertreter (mit entsprechender } \\
\text { Funktion) } \\
\text { Distribution: mit oder an Stelle eines } \\
\text { Substantivs } \\
\end{array}$ & $\begin{array}{l}\text { Verweis, nähere } \\
\text { Bestimmung }\end{array}$ \\
\hline $\begin{array}{l}\text { Partikeln: } \\
\text {-Adverb }\end{array}$ & nicht flektierbar & $\begin{array}{l}\text { Funktion: Attribut oder } \\
\text { Umstandsangabe } \\
\text { Distribution: mit Substantiv, Adjektiv, } \\
\text { Verb }\end{array}$ & $\begin{array}{l}\text { Nähere } \\
\text { Umstände }\end{array}$ \\
\hline -Präposition & nicht flektierbar & $\begin{array}{l}\text { Funktion: Präpositionalkasus } \\
\text { Distribution: vor Substantiven } \\
\text { (Pronomen) }\end{array}$ & $\begin{array}{l}\text { Verhältnisse, } \\
\text { Beziehungen }\end{array}$ \\
\hline -Konjunktion & nicht flektierbar & $\begin{array}{l}\text { Funktion: Verbindung, Einleitung, } \\
\text { Unterordnung } \\
\text { Distribution: zwischen Sätzen, } \\
\text { innerhalb von Satzgliedern und } \\
\text { Attributen }\end{array}$ & $\begin{array}{l}\text { Verknüpfung im } \\
\text { logischen, } \\
\text { zeitlichen, } \\
\text { begründenden, } \\
\text { modalen u. ä. } \\
\text { Sinn }\end{array}$ \\
\hline Interjektion & nicht flektierbar & $\begin{array}{l}\text { gewöhnlich syntaktisch } \text { isoliert; } \\
\text { dialogsteuernde und } \\
\text { Funktion }\end{array}$ & $\begin{array}{l}\text { Empfindungen, } \\
\text { Gefühle, } \\
\text { Stellungnahmen }\end{array}$ \\
\hline
\end{tabular}

Tabla 1 
Fundamental es en este caso subrayar la relación que el autor establece entre la forma de la expresión con esa idea o concepto a la que hace alusión, y recordar la conexión de dicha idea (aunque no venga expresada en la definición) con la realidad extralingüística. Por otro lado, Tesnière define las palabras vacías del siguiente modo:

"Leere Wörter nennen wir die ohne semantische Funktion. Sie sind grammatische Hilfsmittel, deren Aufgabe einzig darin besteht, die Kategorie der vollen Wörter anzugeben, zu präzisieren oder auch zu ändern und die Beziehungen zwischen vollen Wörtern zu regeln." (Tesnière 1980: 64).

Palabras vacías son para el autor conjunciones, preposiciones, pronombres relativos, artículos y los verbos auxiliares de la gramática tradicional (en su terminología juntores y translatores, cf. Tesnière 1980: 81-82). Ahora es primordial destacar cómo Tesnière niega la función semántica de estas palabras, negación que conlleva, según mi apreciación, la negación de la existencia de esa idea o concepto que se relaciona con el significante. De este modo, ese significado gramatical o relacional parece circunscribirse al interior de la lengua, que utiliza estas palabras como elementos internos para su propia organización. El mismo autor les concede no obstante un " (...) völlig abstrakten Gehalt (...)" (Tesnière 1980: 80), que en todo caso no puede equipararse a lo que se entiende por significado.

No sólo en Tesnière se encuentra el intento de distinguir dos clases principales de palabras según su significado: generalmente se distingue un significado directo, inmediato frente a un significado mediato, dependiente de otras palabras; palabras llenas de significado, autosemánticas, frente a palabras de significado funcional, estructural o gramatical; un significado léxico frente a un significado gramatical. En general, sustantivos, adjetivos y verbos son las clases léxicas llenas de significado (así en Duden, que los llama Hauptwortarten, cf. Duden 1984: 89. Tesnière también incluye a los adverbios en esta categoría). La negación del significado en las palabras vacías es un extremo al que de todos modos no suelen llegar otros autores, pero sí a diferenciar tipos de significado como se veía arriba. Este rasgo semántico en las clasificaciones de las clases léxicas se muestra, sin embargo, realmente problemático, como afirma U. Engel:

"Das semantische Verfahren unterstellt, daß jeder Wortklasse per se eine bestimmte Bedeutung zukomme: Nomina bezeichnen Dinge (auch gedachte Dinge), Verben Vorgänge und Zustände, (...). Nimmt man aber andere Beispiele, so wird die Brüchigkeit der hier verwendete Prinzipien deutlich." (Engel 1988: 17).

Hay por ello autores que prefieren en sus gramáticas establecer otros criterios como centrales, v. gr. el sintáctico en G. Helbig y J. Buscha, quienes no obstante reconocen que parte de las clases léxicas poseen rasgos añadidos morfológicos y semánticos. Así, a pesar de estos intentos de categorización a partir de la sintaxis, estos autores establecen también dos clases básicas en las que se hace patente la intervención del plano semántico:

"Von den Wortklassen werden zunächst die vier hauptsächlichen Wortklassen (Verb, Substantiv, Adjektiv, Adverb) dargestellt. Danach folgen die 'Funktionswörter': Diese unterscheiden sich von den zuerst genannten Haupt-Wortklassen dadurch, daß ihre Elemente im wesentlichen grammatische Funktionen ausüben, (...)" (Helbig/Buscha 1989: 21). 
De esta manera, queda establecido que habitualmente se distingue entre palabras llenas de significado y palabras que, si no vacías, poseen como máximo un significado o casi más bien una función relacional, estructural o gramatical.

\section{3 ¿Transformación semántica de palabras vacías?}

3.3.1 Aceptando este hecho, FR como nicht (so) ohne sein en alemán o ponerle peros a alguien/algo en español se alimentan de palabras vacías, de elementos intralingüísticos sin relación con la realidad extralingüística, esto es, se alimentan de la propia lengua. La evidencia de que tanto el componente del FR ohne como el componente pero adquieren un significado translaticio, fácilmente reconocible por la estructura composicional de los FR reflejada en las definiciones de los diccionarios correspondientes ( $\rightarrow$ "ponerle reparos a alguien/algo", $\rightarrow$ "nicht so harmlos sein, wie man annehmen könnte"), exige al menos una revisión parcial de la afirmación de una inexistencia de significado o un significado menor gramatical de naturaleza diversa en estas clases léxicas. Es decir, si las palabras pero y ohne son preposiciones sin función semántica o una función reducida, ¿cómo pueden sufrir una transformación de significado metafórica, basada en la similitud con otro concepto? ¿Es por tanto el significado de las conjunciones pero y ohne de naturaleza tan diversa a la de substantivos o verbos o es similar, ya que desde un punto de vista cognitivo funcionan igual?. Relevante es también que la teoría general sobre fraseología detecta en estos FR irregularidades gramaticales (en estos casos, v. gr., las conjunciones cumplen funciones habituales de los substantivos y los adjetivos), rasgo general relativo a la estabilidad de las unidades fraseológicas (cf. Fleischer 1982: 53). Con ello parece confirmarse la validez de los rasgos formales que definen las clases léxicas, pero queda clara la dificultad de aplicar rasgos semánticos distintivos a cada una de ellas, como indica Lutzeier:

"Die einzige Möglichkeit, wie wir diese Ergebnisse"13 verwerten können, ist die Ablehnung jeglicher klaren Unterscheidung zwischen Vollwörtern und Strukturwörtern. (...)." "Es gibt also keine klar voneinander unterschiedene Arten von Bedeutungen, die wir für bestimmte Wörter reservieren müssen; alle Wörter haben vielmehr dieselbe Art von Bedeutung." (Lutzeier 1985: 24, 26).

Esta exposición pretende proporcionar un punto más de apoyo a la opinión de Lutzeier por medio del análisis de unidades fraseológico-idiomáticas. No pretende, no obstante, establecer la imposibilidad de determinar rasgos semánticos en cada clase léxica, pero sí hacer ver la dificultad de realizar una separación tajante. Indudable permanece en todo caso el interés por investigar posibles particularidades en los significados de las distintas clases léxicas, en su grado de abstracción y en la gradación que se puede establecer en su relación más o menos mediata con la realidad circundante. ${ }^{14}$

\footnotetext{
${ }^{13}$ Aquí Lutzeier no se refiere a los resultados expuestos en este artículo, pero sí a otras observaciones de tipo semántico.

${ }^{14}$ Así, v. gr., no se puede obviar la existencia de algunas propiedades semánticas más o menos adscribibles a alguna clase léxica, como es el hecho de que existan realmente conjunciones sin significado, tal y como muestra la clasificación de Eisenberg en conjunciones temporales, condicionales, finales, causales, concesivas, consecutivas, instrumentales, comparativas y vacías de contenido (Eisenberg 1986: 311).
} 
3.3.2 De este modo, la hipótesis establecida como punto de partida para el análisis de estos FR no se constata, por lo que no puede afirmarse que estas unidades fraseológicas se alimenten del propio sistema lingüístico, como sucedía en todos los casos de los anteriores apartados.

En cualquier manera, contrastivamente se observa la existencia de FR formados a partir de irregularidades gramaticales ligadas a las clases léxicas no principales en ambos idiomas, incluso con alguna equivalencia individual. Ello refuerza la suposición de que la tesis de Lutzeier es aplicable a principios gramaticales generales.

FR con preposiciones como componentes con significado "pleno" son los pros y los contras, das Für und Wider, bei jm. aus und ein sein, weder aus noch ein wissen.

Se hallan igualmente FR formados con conjunciones (como los ejemplos anteriormente utilizados), v. gr., no hay pero que valga o Wenn und Aber.

Por último, se verifican formas pronominales como componentes internos (incluso a veces pertenecientes a otro sistema lingüístico) que posiblemente necesiten un análisis más detallado y específico, v. gr., mein und dein verwechseln/nicht unterscheiden können, (mit jm.) per du sein, ser el quid de la cuestión, tener u. p./u. c. su aquel, hablarse de tú, caerse u. c. de suyo.

En definitiva, estos FR demuestran que en unidades pertenecientes a clases léxicas como las preposiciones, las conjunciones y los pronombres puede encontrarse un sustrato de significado común con las clases de palabras principales, que hace problemática la distinción inequívoca de las clases léxicas en el plano semántico.

\section{Bibliografia}

-H. Burger, “die achseln zucken” - Zur sprachlichen kodierung nicht-sprachlicher kommunikation", Wirkendes Wort 26 (1976) 311-334.

-F. Daneš, "The Relation of Centre and Periphery as a Language Universal", Travaux linguistiques de Prague 2 (1966) 9-21.

-D. Dobrovol'skij, Kognitive Aspekte der Idiom-Semantik. Studien zum Thesaurus deutscher Idiome (Tubinga 1995).

-Duden. Grammatik der deutschen Sprache (Mannheim/Viena/Zürich 1984).

-P. Eisenberg, Grundriss der deutschen Grammatik (Stuttgart 1986).

-W. Eismann, "Zeichenbausteine als Zeichen. Das Alphabet in der Phraseologie", Aktuelle Probleme der Phraseologie. Symposium 27.-29.9.1984 in Zürich (Berna 1987) 225-244.

-U. Engel, Deutsche Grammatik (Heidelberg 1988). 
-A. Eschbach, "Semiotik", Lexikon der germanistischen Linguistik (Tubinga 1980) 4157

-W. Fleischer, Phraseologie der deutschen Gegenwartssprache (Leipzig 1982).

-C. Földes, "Anthroponyme als Strukturkomponenten deutscher Phraseologismen", Zeitschrift für germanistische Linguistik 15 (1987) 1-19.

-C. Földes, "Phraseologie und Landeskunde - am Material des Deutschen und des Ungarischen", Zielsprache Deutsch 21 (1990) 11-15.

-J. Häusermann, Phraseologie. Hauptprobleme der deutschen Phraseologie auf der Basis sowjetischer Forschungergebnisse (Tubinga 1977).

-G. Helbig/J. Buscha, Deutsche Grammatik. Ein Handbuch für den Ausländerunterricht (Leipzig 1989).

-Z. Klimaszewska, "Die Phraseologie auf kulturhistorischem Hintergrund am Beispiel des Deutschen, Niederländischen und Polnischen", Akten des VIII Internationalen Germanistenkongresses (Munich 1991), t. 4º, 362-369.

-J. Korhonen (ed.), Studien zur Phraseologie des Deutschen und des Finnischen (Bochum 1995, 1996), 2 t.

-J. P. Larreta, "Eufemismos y tabúes lingüísticos en la fraseología alemana”, mAGAzin 1 (1996) 56-61.

-P. R. Lutzeier, Linguistische Semantik (Stuttgart 1985).

-C. K. Ogden/I. A. Richards, Die Bedeutung der Bedeutung (Fráncfort d. M. 1974).

-Ch. S. Peirce, Phänomen und Logik der Zeichen (Fráncfort d. M. 1993).

-B. A. Serébrennikov (ed.), Allgemeine Sprachwissenschaft (Munich/Salzburgo 1973).

-L. Tesnière, Grundzüge der strukturellen Syntax (Stuttgart 1980).

\section{Diccionarios}

-Duden. Redewendungen und sprichwörtliche Redensarten. Idiomatisches Wörterbuch der deutschen Sprache (Mannheim/Leipzig/Viena/Zürich 1994).

-J. M. Iribarren, El porqué de los dichos. Sentido, origen y anécdota de los dichos. Modismos y frases proverbiales de España con otras muchas curiosidades (Pamplona 1994).

-L. Röhrich, Das große Lexikon der sprichwörtlichen Redensarten (Friburgo/Basilea/Viena 1991).

-F. Varela/H. Kubarth, Diccionario fraseológico del español moderno (Madrid 1994). 